\title{
VÍNCULOS PARENTALES EN LOS HOGARES DE LOS ESTUDIANTES DEL QUINTO DE SECUNDARIA, INSTITUCIÓN EDUCATIVA JOSÉ FAUSTINO SÁNCHEZ CARRIÓN, SAYÁN, 2018
}

\author{
PARENTAL LINKS IN THE HOUSEHOLDS OF THE STUDENTS OF THE FIFTH SECONDARY \\ SCHOOL, INSTITUCIÓN EDUCATIVA JOSÉ FAUSTINO SÁNCHEZ CARRIÓN, SAYÁN, 2018
}

Yuleisi Milagros Yarlaqué Román ${ }^{1}$, Iris Yajaira Calle Villavicencio ${ }^{1}$,

Margot Albina Castillo Alva ${ }^{2}$, Luz Amparo Bernal Villarreal ${ }^{2}$, Cesar Alexander López Ramos ${ }^{3}$

\section{RESUMEN}

Objetivo: Determinar el nivel y tipos de vínculos parentales que se presentan en los hogares de los estudiantes del quinto de secundaria de la Institución Educativa José Faustino Sánchez Carrión, Sayán 2018. Material y Métodos: El diseño de la investigación es no experimental, que nos permitió determinar las características del problema sin alterarlas o cambiarlas. El tipo de investigación utilizado según la finalidad es de tipo básica, descriptiva de corte transversal, debido a que se apoyó primeramente en el contexto teórico, buscando ampliar y profundizar el caudal de conocimiento científico existentes acerca de la realidad. Resultado: Se Obtuvo un porcentaje alto de $41,2 \%$ en el vínculo de constricción cariñosa materno y 42,6\% en el vínculo de constricción cariñosa paterno. Conclusión: El tipo de vinculo que predominó fue el de contricción cariñosa, que se caracteriza por padres con alto cuidado y sobreprotección para con sus hijos, haciendoles vulnerables a diversas situaciones.

Palabras clave: Vínculos Parentales; Cuidado; Sobreprotección.

\section{ABSTRACT}

Objective: Determine the level of parental links that are present in the homes of the students of the fifth year of high school of the José Faustino Sánchez Carrión, Sayán 2018 Educational Institution. Material and Methods: The design of the research is not experimental, which allowed us to determine the characteristics of the problem without altering or changing them. The type of research used according to the purpose is of a basic, descriptive cross-sectional type, because it was based primarily on the theoretical context, seeking to broaden and deepen the flow of existing scientific knowledge about reality. Result: A high percentage of $41.2 \%$ was obtained in the bond of affectionate maternal constriction and $42.6 \%$ in the bond of paternal affectionate constriction. Conclusions: The type of hond that predominated was that of affectionate constriction that is characterizad by parents wits high care and over protection for heir children, walking thens vulnerable to various situationes.

Key words: Parental Links; Care; Overprotection.

\footnotetext{
'Egresada de la E.P. de Trabajo Social de la Universidad Nacional José Faustino Sánchez Carrión de Huacho (Huacho - Perú). Email: yuleisi_yarlaque@hotmail.com

${ }^{2}$ Docentes de la E.P. de Trabajo Social de la Universidad Nacional José Faustino Sánchez Carrión de Huacho (Huacho - Perú)

${ }^{3}$ Universidad Nacional José Faustino Sánchez Carrión de Huacho (Huacho - Perú)
}

Recibido:03/03/18 Aprobado:12/06/18 


\section{INTRODUCCIÓN}

Los seres humanos establecen una infinidad de relaciones significativas con otros en el transcurso de la vida, pero sin duda los padres ejercen una influencia determinante en el desarrollo psicológico del individuo. Por ello muchos teóricos se han interesado en conocer los procesos que tienen lugar en la relación padres - hijos y en explicar cómo éstas pueden influir en los comportamientos y el desarrollo de la personalidad.

Desde el momento en que el hijo es concebido se forja una relación donde la vida del nuevo ser depende principalmente de la madre, y donde gradualmente el niño a medida que se desarrolla se va haciendo más independiente y autónomo en un proceso largo y complejo. Muchos factores intervienen en este proceso siendo el vínculo entre los padres y sus hijos uno de los más importantes, ya que generan relaciones sanas y seguras. El tipo de relación que establece el niño y su madre no sólo es significativo en la infancia, sino también en el curso de todo.

Vivimos en una sociedad en la que los hijos no se planean, y hace que, el ser padre o madre sea improvisado y en muchas ocasiones se prefiera abandonar o dejar de lado a los niños, es aquí donde se presentan los problemas relacionados con los vínculos que se han establecido.

Lo que sucede cuando no se cumplen aquellas funciones y aquel niño crece con un vínculo, débil e inseguro, Zorrilla (2017). Menciona que; "La calidad de los cuidados parentales que un niño recibe en sus primeros años es de vital importancia para el futuro de la salud mental, o dicho de otra manera para que un niño o niña pequeña se desarrolle de una manera óptima se requiere poder establecer una relación cálida, íntima y continuada con alguna figura parental, sea con la madre, el padre u otro sustituto parental", Oliva (2006) menciona que muchos problemas adolescentes se deben a la falta de apoyo y afecto, lo cual habla del desamparo en torno a las figuras parentales.

Como se puede apreciar las figuras parentales son esenciales, su presencia o ausencia genera una huella casi imposible de reemplazar, en su camino en el desarrollo y en la construcción de la personalidad.

La forma en que el niño o niña se relacionará en el futuro, la forma de resolver problemas, y los modelos para generar sus propia identidad se asocia a la figura de los padres y los lazos afectivos que pueden desarrollarse a lo largo de su vida, es importante que los padres se mantengan informados y así conocer cómo abordar determinadas situaciones de amenaza, teniendo en cuenta que la familia es el primer lugar donde el niño se socializa, sin dejar de lado la escuela, la cual constituye el segundo agente de socialización vital para el desarrollo del niño y su personalidad.

Según investigaciones en América Latina las ausencias de vínculos parentales afectan su desarrollo emocional, manifestando conductas y acciones inadecuadas, como por ejemplo el pandillaje, la drogadicción, el narcotráfico etc., estos problemas se evidencian cuando uno de los hijos sufren la ausencia repentina de afecto por parte de los padres y empiezan a tener una imagen pobre de sí mismo trayendo como consecuencia el resentimiento hacia el mundo y tomando cierta dependencia emocional por miedo al abandono ante la ruptura del vínculo el buscará establecer una relación con cualquier persona que se acerque, sustituyendo los lazos afectivos que tenía con los padres, es esta etapa la más difícil pues el adolescente observa a su alrededor familias vinculadas, con mejores relaciones y a la vez familias que podrían ser similares a la suya, ansía dialogar con alguien lo que está sintiendo, llenar aquellos vacíos, estando propensos a cometer actos ilícitos o actos en contra de su propia vida.

En los estudiantes del nivel secundario de la I.E José Faustino Sánchez Carrión existen diferentes tipos de familia, en las cuales el vínculo parental ha sido fuertemente establecido mediante el afecto y cuidado, mientras que en otros este vínculo está débil o ausente, ya sea por la falta de comunicación en el hogar, divorcios de los padres, enfermedades o pérdidas de la figura parental, son estos los que afectan emocionalmente el desarrollo del niño por otro lado un niño que se beneficia de una relación saludable con ambos estará preparado para afrontar problemas y lograr un buen desarrollo.

Con la siguiente investigación queremos explicar que resulta fundamental crear conciencia en los padres la necesidad de generar espacios que faciliten una vinculación estable y de calidad con los hijos. Esto permitirá a los padres conocer de mejor manera a sus hijos y por ende, podrán detectar las conductas de riesgo que son propias de la etapa adolescente, así como también percibir cambios en 
los estados anímicos que pueden ser síntomas de depresión o de otros problemas. Del mismo modo, entregarán a sus hijos la certeza de que ambos padres estarán ahí cuando los necesiten, brindando al mismo tiempo una base segura que garantizará un adecuado desarrollo psicológico y protección frente a situaciones amenazantes.

Conforme a lo expuesto en los párrafos precedentes consideramos de mucha importancia desarrollar la presente investigación.

Nuestra investigación se enfocó en los recuerdos de infancia de los adolescentes de la institución educativa José Faustino Sánchez Carrión para medir su nivel de vínculo que se ha establecido hasta la actualidad con cada padre.

\section{MATERIALES Y MÉTODOS}

El diseño de la investigación es no experimental, que nos permitió determinar las características del problema sin alterarlas o cambiarlas. El tipo de investigación a utilizarse según la finalidad es de tipo básica, descriptiva,

Así mismo se realizó la investigación haciendo uso del enfoque cuantitativo. La investigación es de enfoque cuantitativo, es decir se midió la existencia de la variable en la población a través de la aplicación de instrumentos de recolección de datos, el cual permitió cuantificar los datos recogidos en la población de estudio, (Hernández, Fernandez y Bautista, 2010). Población: La población está conformada por 250 estudiantes del nivel secundaria de la Institución Educativa José Faustino Sánchez Carrión. Muestra: La muestra estuvo conformada por 70 estudiantes que cursan el quinto año de secundaria, de ambos sexos los cuales se encuentran entre las edades de 15 a 17 años.

En las técnicas para el procesamiento de información se tomo:

*Análisis Documental

Mediante el análisis documental y sus respectivos instrumentos, se revisaron fuentes bibliográficas, publicaciones especializadas y portales de internet directamente relacionados con la variable de estudio.

A través de la aplicación del instrumento de Vínculos Parentales del autor Parker, Tupling y Brown (1979) se recopiló información como respuesta al objetivo general.

Mediante la técnica de la observación se pudo identificar los comportamientos y las conductas de cada alumno de la población a estudiar, lo cual nos servirá para desarrollar nuestra investigación con la variable Vínculos parentales.

*Ficha Técnica de Instrumentos

El instrumento de vínculos parentales estuvo constituido por 25 ítems , 13 ítems pertenecientes a la dimensión de Cuidado y 12 para la dimensión sobreprotección, con una escala de Likert de 0-3, en donde muy de acuerdo es igual a 0 puntos, de acuerdo $=1$ punto, en desacuerdo $=2$ puntos y muy en desacuerdo $=3$ punto)los cuales se aplican a los estudiantes sobre la percepción que tienen sobre su padre y madre por separado.

*Administración de los instrumentos y obtención de los datos

Para el acopio de la información se aplicó el Instrumento de vínculos parentales, confiable y validado en la investigación de Melis (2001), como medida de la consistencia interna del test, se calculó el coeficiente de correlación Spearman Brown y el coeficiente Alfa de Crombach. Los índices para la versión estandarizada se encuentran entre los valores de 0,78 y 0,89 , respectivamente, para las escalas subdivididas por el sexo del progenitor. Por otra parte, se calculó el coeficiente de correlación de Pearson, para determinar la relación entre las dimensiones cuidado y sobreprotección, donde los resultados oscilaron entre $-0,190$ y $-0,368$, por lo cual es posible concluir que posee un adecuado índice de confiabilidad.

*Análisis Estadístico

Se llevó a cabo utilizando el paquete estadístico SPSS 23.0 el cual procesaron los resultados y lograron la interpretación, análisis y discusión de la respuesta al objetivo de la investigación, así mismo contar con las conclusiones, implicando el objetivo que será el producto final de la investigación.

\section{RESULTADO}

Tabla 1. Nivel de vínculos parentales

\begin{tabular}{ccccc}
\hline & \multicolumn{2}{c}{ Madre } & \multicolumn{2}{c}{ Padre } \\
\cline { 2 - 5 } Tipo de vínculo parental & $\mathbf{F}$ & $\mathbf{\%}$ & $\mathbf{F}$ & $\mathbf{\%}$ \\
\hline Vínculo Óptimo & 23 & 33,8 & 19 & 27,9 \\
Control sin afecto & 9 & 13,2 & 11 & 16,2 \\
Constricción Cariñosa & 28 & 41,2 & 29 & 42,6 \\
Vínculo Ausente o débil & 8 & 11,8 & 9 & 13,2 \\
Total & 68 & 100,0 & 68 & 100,0 \\
\hline
\end{tabular}

Parker et al (1979), argumentan que: Un vínculo parental, se entiende como una conducta de apego que tiene como resultado el logro o la conservación de la proximidad con otro individuo claramente identificado al que se considera mejor capacitado 
para enfrentarse al mundo (padre o madre), señalando la más idónea, aquella relacionada con la alta presencia de cuidado y baja presencia de sobreprotección, o vinculo óptimo, que se caracteriza por ser afectuoso, empático y, a su vez, favorece la independencia y la autonomía.

Sin embargo, en los datos obtenidos relacionado a las actitudes de la madre se observa que el porcentaje valido más alto lo obtuvo el vinculo de constricción cariñosa con un porcentaje de $41,2 \%$ lo cual se explica por tener un alto nivel de cuidado y alto el nivel de sobreprotección, por otro lado se percibe un nivel bajo en el vínculo óptimo con un porcentaje de $33,8 \%$.

Así mismo observamos en las actitudes relacionada al padre que el porcentaje válido más alto lo obtuvo el vínculo de constricción cariñosa los cuales se caracterizan por presentar afectuosidad, contención emocional, empatía y cercanía, por un lado, y al mismo tiempo son controladores, intrusivos, tienen un contacto excesivo, infantilizan y previenen la conducta autónoma de sus hijos con un porcentaje de $42,6 \%$, seguidamente podemos observar un porcentaje bajo en el vínculo óptimo el cual debería ser el más adecuado para cada familia con un porcentajes de $27,9 \%$ en relación al vínculo parental paterno.

\section{DISCUSIÓN:}

La investigación Vínculos Parentales en los hogares de los estudiantes de la Institución Educativa José Faustino Sánchez Carrión - Sayán 2018, tuvo por objetivo determinar el nivel de los vínculos parentales que se presentan en los hogares de los estudiantes, para poder evaluar los niveles se utiliza cuatro tipos de vínculos parentales, se obtuvo como resultado que el vínculo constricción cariñosa tuvo un nivel alto con un $41,2 \%$ para madre y un $42,6 \%$ para padre, en segundo lugar el vínculo óptimo con un $33,8 \%$ para madre y 27,9 para padre , en tercer lugar el vínculo de control sin afecto con un porcentaje de $13,2 \%$ para madre y $16,2 \%$ para padre , así mismo el nivel más bajo lo obtuvo el vínculo ausente o débil con un 11,8\% para madre y 13,2\% para padre. Estos resultados se asemejan a la investigación realizada por (Amezquita, M. 2013) titulada Validez del constructo del parental Bonding en una muestra de adolescentes del quinto de secundaria de un colegio público de Lima Metropolitana cuyo objetivo fue analizar la validez del constructo del PBI en nuestro contexto cultural y en población adolescente, en la cual el vínculo de constricción cariñosa se encuentra en un porcentaje de $30 \%$.

Finalmente se puede concluir lo siguiente: De los 70 estudiantes evaluados que representan el $100 \%$ se observa que el $41,2 \%$ y $42,6 \%$ tanto para madres como padres respectivamente poseen el vínculo de constricción cariñosa alto, en segundo lugar para el vínculo óptimo se obtuvo un porcentaje de 33,8\% para madre y 27,9 para padre , en tercer lugar el vínculo de control sin afecto con un porcentaje de $13,2 \%$ para madre y $16,2 \%$ para padre, así mismo el nivel más bajo lo obtuvo el vínculo ausente o débil con un $11,8 \%$ para madre y $13,2 \%$ para padre, entonces podemos decir que el tipo de vínculo que predomina es el vínculo de constricción cariñosa el cual se caracteriza por padres que poseen alto cuidado y alta sobreprotección para con sus hijos, haciendo de ellos jóvenes vulnerables a situaciones amenazantes, relaciones sociales débiles, inseguridad para tomar decisiones, etc., lo cual se observara en el futuro de estos jóvenes.

\section{REFERENCIABIBLIOGRÁFICA}

Zorrilla, B.A. (2017). Apoyo y vinculo: Conergencias y Divergencias. Area 3. Cuadernos de temas grupales e institucionales. ISSN 1886-6530. $N^{\circ} 21$.

Oliva, A. (2006). Relaciones familiares y desarrollo adolescente. Anuario de Psicología. 37(3): 209-223.

Mezquita,V.M.K. (2013). Validez de cosntricto del parental Bonding Instruments en una muestra de adolescentes de 5to de secundaria de un colegio público de Lima Metropolitana. Tesis para optar titulo de licenciado en Psicología con mención en Psicología clínica. Facultad de Letras y Ciencias Humanas. Pontificia Universidad Católica del Perú.

Hernandez, S.R., Fernandez, C.C. \& Baptista, L.P. (2010). Metodología de la investigación. Editorial Mc. Graw Hill. 5ta Edición.

Melis F, Davila, M.A., Ormeño, V., Vera, V. Greppi, C.\& Gloger, S. (2001). Estandarización del P.B.I. (Parental Bonding Instrument), versión adaptada a la población entre 16 y 64 años del Gran Santiago. Rev. Chil. Neuro- Psiquiat. 39 (2): 132-139.

Parker, G. Tupling, H. \&Brown, L.B. (1979). A parental Bonding Instrument. British Journal of Medical Psychology. 52 (1): 1-10. 